\title{
Correction to: An integrative phylogenomic approach to elucidate the evolutionary history and divergence times of Neuropterida (Insecta: Holometabola)
}

\author{
Alexandros Vasilikopoulos ${ }^{1{ }^{*}+}$, Bernhard Misof ${ }^{1{ }^{*}+}$, Karen Meusemann ${ }^{1,2,3}$, Doria Lieberz ${ }^{1}$, Tomáš Flouri ${ }^{4}$, \\ Rolf G. Beutel ${ }^{5}$, Oliver Niehuis ${ }^{2}$, Torsten Wappler ${ }^{6}$, Jes Rust ${ }^{7}$, Ralph S. Peters ${ }^{8}$, Alexander Donath ${ }^{1}$, \\ Lars Podsiadlowski ${ }^{1}$, Christoph Mayer ${ }^{1}$, Daniela Bartel ${ }^{9}$, Alexander Böhm ${ }^{9}$, Shanlin Liu ${ }^{10}$, Paschalia Kapli ${ }^{4}$, \\ Carola Greve ${ }^{11}$, James E. Jepson ${ }^{12}$, Xingyue Liu' ${ }^{10}$, Xin Zhou ${ }^{10}$, Horst Aspöck ${ }^{13}$ and Ulrike Aspöck ${ }^{9,14}$
}

\section{Correction to: BMC Evol Biol 20, 64 (2020) \\ https://doi.org/10.1186/s12862-020-01631-6}

Following publication of the original article [1], the authors discovered that some pie charts had been misplaced in the tree of Fig. 2a, and in the trees of supplementary figures S16, S22, S24 (Additional file 3) due to incorrect visualization of the output of ASTRAL [2]. These quartet support values are, however, correctly provided in supplementary tables S16-S19 of the original publication. Thus, the original publication is inconsistent on a couple of support values for specific phylogenetic relationships. We provide now a corrected Fig. 2 and an updated Additional file 3 with corrected Figures S16, S22, S24. The changes in Fig. 2a do not affect the controversial branches discussed in the original publication (e.g. relationships within Osmyloidea, phylogenetic affinities of Chrysopidae and Hemerobiidae, monophyly of Myrmeleontiformia and Myrmeleontidae). The corrected Fig. 2a shows the correct (increased) quartet support for the monophyly of

The original article can be found online at https://doi.org/10.1186/s12862 020-01631-6.

* Correspondence: alexvasilikop@gmail.com; b.misof@leibniz-zfmk.de ${ }^{+}$Alexandros Vasilikopoulos and Bernhard Misof contributed equally to this work.

${ }^{1}$ Centre for Molecular Biodiversity Research, Zoological Research Museum Alexander Koenig, 53113 Bonn, Germany

Full list of author information is available at the end of the article
Neuropterida $(\mathrm{q} 1=0.798, \mathrm{q} 2=0.098, \mathrm{q} 3=0.103)$ and the correct (lower) quartet support for the monophyly of Neuroptera ( $\mathrm{q} 1=0.476, \mathrm{q} 2=0.168, \mathrm{q} 3=0.357$ ). Quartet support for the monophyly of Neuroptera (q1) is still higher in comparison to the other two topologies ( $\mathrm{q} 2$, q3) around this specific internode. Therefore these changes do not alter the conclusions of [1]. The authors would like to apologize for any inconvenience caused.

\section{Abbreviations}

ACSR: Ancestral character state reconstruction; FCLM: Four-cluster likelihood mapping; SRH: Global stationary (time-) reversible and homogeneous conditions

\section{Supplementary information}

Supplementary information accompanies this paper at https://doi.org/10. 1186/s12862-020-01695-4.

Additional file 3: Supplementary Figures S1-S56. The supplementary
figures include: 1) all phylogenetic trees inferred from the analyses of different
datasets and tree-inference methods, 2) results of additional ACSR analyses
under different parameters, 3) heatmaps visualizing the pairwise alignment
completeness scores of all analyzed supermatrices, 4) heatmaps visualizing the
pairwise deviation from SRH conditions in each analyzed supermatrix, 5) scat-
ter plot of the mean posterior node-age estimates from run 1 plotted against
the mean posterior node-age estimates from run 2 when using all fossil cali-
brations, 6) beanplots of median posterior node-age estimates from run 1 and
from run 2 when using all fossil calibrations, 7) scatter plots of the mean pos-
terior node-age estimates plotted against the 95\% higher posterior density Cl-
width of each node when running the dating analyses with or without data.




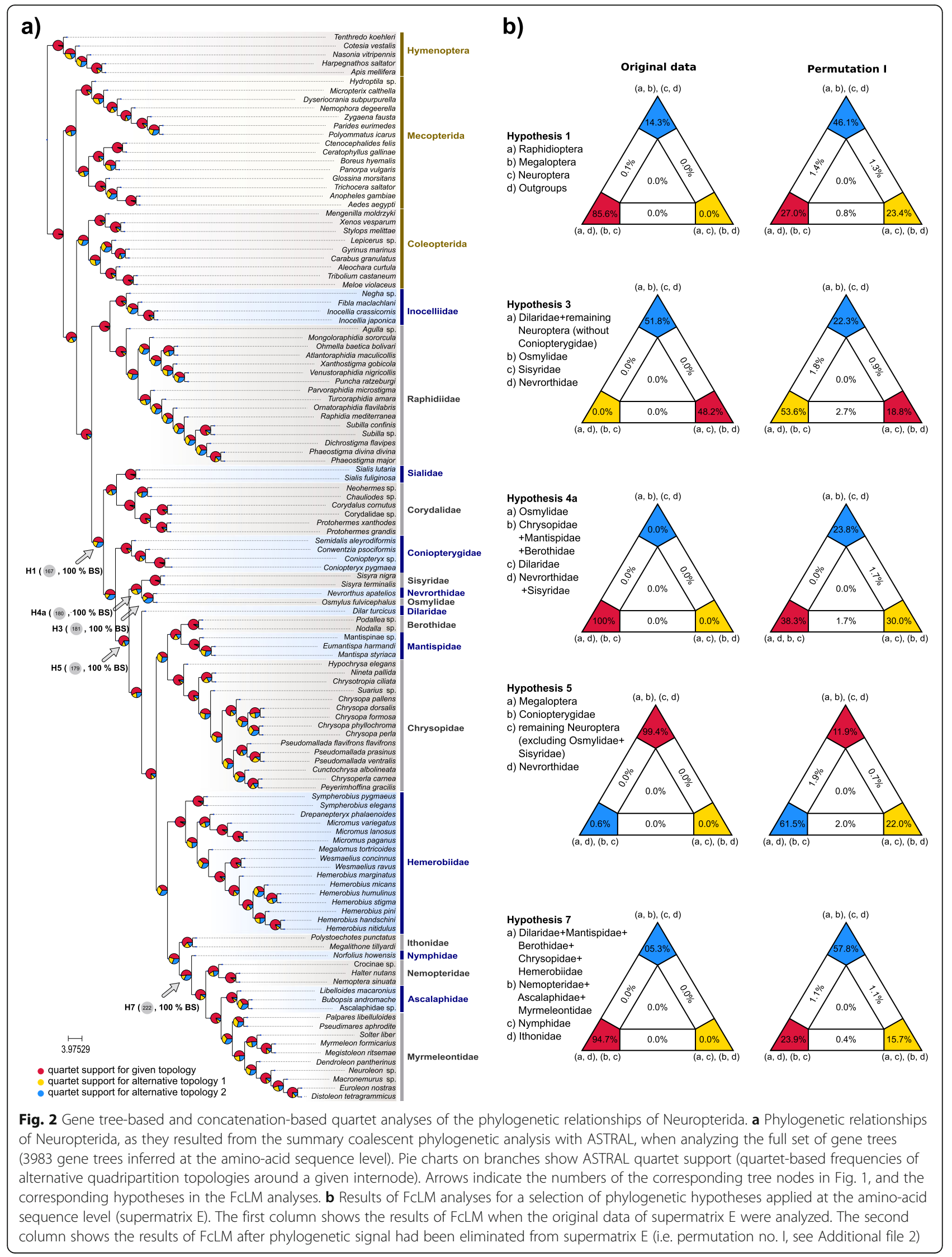




\section{Author details}

${ }^{1}$ Centre for Molecular Biodiversity Research, Zoological Research Museum Alexander Koenig, 53113 Bonn, Germany. ${ }^{2}$ Department of Evolutionary Biology and Ecology, Institute of Biology I (Zoology),

Albert-Ludwigs-Universität Freiburg, 79104 Freiburg, Germany. ${ }^{3}$ Australian National Insect Collection, National Research Collections Australia,

Commonwealth Scientific and Industrial Research Organisation (CSIRO),

Canberra ACT 2601, Australia. ${ }^{4}$ Department of Genetics, Evolution and Environment, University College London, London WC1E 6BT, UK. Institut für Zoologie und Evolutionsforschung, Friedrich-Schiller-Universität Jena, 07743 Jena, Germany. ${ }^{6}$ Natural History Department, Hessisches Landesmuseum Darmstadt, 64283 Darmstadt, Germany. ${ }^{7}$ Steinmann-Institut für Geologie, Mineralogie und Paläontologie, Rheinische Friedrich-Wilhelms-Universität Bonn, 53115 Bonn, Germany. ${ }^{8}$ Centre for Taxonomy and Evolutionary Research, Arthropoda Department, Zoological Research Museum Alexander Koenig, 53113 Bonn, Germany. ${ }^{9}$ Department of Evolutionary Biology, University of Vienna, 1090 Vienna, Austria. ${ }^{10}$ Department of Entomology, China Agricultural University, 100193 Beijing, People's Republic of China. "LOEWE Centre for Translational Biodiversity Genomics (LOEWE-TBG), 60325 Frankfurt, Germany. ${ }^{12}$ School of Biological, Earth and Environmental Sciences, University College Cork, Distillery Fields, North Mall, T23 N73K Cork, Ireland. ${ }^{13}$ Institute of Specific Prophylaxis and Tropical Medicine, Medical

Parasitology, Medical University of Vienna (MUW), 1090 Vienna, Austria,

${ }^{14}$ Zoological Department II, Natural History Museum of Vienna, 1010 Vienna, Austria.

Published online: 15 October 2020

\section{Reference}

1. Vasilikopoulos A, et al. An integrative phylogenomic approach to elucidate the evolutionary history and divergence times of Neuropterida (Insecta: Holometabola). BMC Evol Biol. 2020;20:64. https://doi.org/10.1186/s12862020-01631-6.

2. Zhang C, et al. ASTRAL-III: Polynomial time species tree reconstruction from partially resolved gene trees. BMC Bioinformatics. BMC Bioinformatics; 2018; 19:15-30. 